\title{
Emerging concepts in glioma biology: implications for clinical protocols and rational treatment strategies
}

\author{
Stephen M. Wiesner, M.T., Ph.D., Andrew Freese, M.D., Ph.D., \\ AND John R. OHLFest, Ph.D. \\ Department of Neurosurgery, University of Minnesota, Minneapolis, Minnesota
}

\begin{abstract}
Glioblastoma multiforme (GBM), the most common primary central nervous system neoplasm, is a complex, heterogeneous disease. The recent identification of stem cells in murine tumor xenografts that were capable of recapitulating the tumor phenotype adds a new dimension of complexity to the already challenging treatment of patients with GBMs. Although specific cellular and genetic changes are commonly associated with GBM, the mechanism by which those changes occur may have a significant impact on treatment outcome. Of the many bioinformatics techniques developed in recent years, gene expression profiling has become a commonly used research tool for investigating tumor characteristics, and the development of rationally targeted molecular therapies has also accelerated following the initial success of specifically designed inhibitors in the treatment of malignancies. Despite these advances in research techniques and targeted molecular therapies, however, limited clinical impact has been achieved in the treatment of infiltrative malignancies such as GBMs. Thus, further extension in survival of patients with GBMs may require use of multiple analyses of tumors to develop tailored therapies that reflect the inter- and intratumoral heterogeneity of this disease. In this review, the authors briefly consider the potential use of expression profiling combined with mutation analysis in the development of treatment modalities to address the heterogeneity of this complex tumor phenotype.
\end{abstract}

KEY WORDS • glioblastoma multiforme • microarray • stem cell • targeted therapy

\section{OVERVIEW}

Glioblastoma multiforme is the most common primary central nervous system neoplasm, accounting for more than half of all such tumors. ${ }^{6,25}$ Despite advances in survival for many patients with malignancies, the prognosis for those with GBM remains poor, even with modern aggressive intervention, including resection, radiotherapy, and chemotherapy. The overall 2-year survival rate is $25 \%$ at best, and 5 -year survival rates remain in the low single digits. ${ }^{25,42}$ Thus, it is clear that new treatments are needed to overcome the limitations of conventional therapy. Emerging evidence indicates that patient-specific therapies tailored to the unique biology of an individual's GBM may be required to achieve significant improvements in clinical outcomes. In this article we briefly discuss emerging insights into glioma cell biology and common genetic mutations. In addition, we highlight how understanding the cell biology and gene expression profiles of each patient's GBM could be useful in implementing more effective treatment.

Abbreviations used in this paper: BTSC = brain tumor stem cell; $\mathrm{EGFR}=$ epidermal growth factor receptor; GBM = glioblastoma multiforme; JAK/STAT = Janus tyrosine kinase/signal transducer and activator of transcription; MAPK = mitogen-activated protein kinase; NSC $=$ neural stem cell; PDGFR $\beta=$ platelet-derived growth factor receptor- $\beta$; PI3K = phosphatidylinositol 3-kinase; $\mathrm{RB}=$ retinoblastoma; $\mathrm{RTK}=$ receptor tyrosine kinase.

\section{ASPECTS OF GBM}

\section{Cellular Characteristics}

The GBM is a diffuse and infiltrative tumor. Migrating GBM cells can be found several centimeters away from the margins of resection. Accordingly, although tumor recurrence predominantly occurs near the primary resection site, it can arise in distant regions of the brain..$^{23,44}$ The GBM is composed of genetically and morphologically diverse cells, not only among different patients, but also among cells within the same tumor. Furthermore, any therapy that relies on cell division to kill tumor cells is complicated by the fact that only a fraction of glioma cells are actively mitotic during a given treatment window. ${ }^{11}$

Mounting evidence indicates that, similarly to leukemia and breast cancer, GBM tumors are composed of stem celllike precursor cells and also more differentiated tumor cells. ${ }^{44,45,51}$ The former, which we will call BTSCs, are endowed with many features of NSCs, including the capacity for self-renewal, the ability to generate daughter cells with different phenotypes from a single mother cell, the capacity to differentiate into a diverse population of cells, and expression of NSC markers. ${ }^{45,51}$ Moreover, BTSCs have been isolated from human GBMs that can form tumors in the brains of mice that bear a morphological resemblance to the 
original human tumor. ${ }^{45}$ These BTSCs demonstrate immunoreactivity for $\mathrm{CD} 133$, a primitive progenitor cell marker, and appear to be the tumor-initiating cells not only in mouse xenografts but also potentially in spontaneous human GBM. Furthermore, when BTSCs were induced to differentiate in vitro, they retained the ability to form neurospheres when cultured in stem cell-supportive media, an ability that is unique to BTSCs. Thus, BTSCs appear to have the capacity to revert from a terminally differentiated state back to a more primitive state, a characteristic distinct from normal NSCs and consistent with cells found in other malignancies. ${ }^{51}$

The ability of cells isolated from human tumors to self renew and contribute to various cell types in both in vitro and in vivo tumorigenesis models establishes a potential mechanism for the complex heterogeneity characteristic of GBM and highlights the need for therapeutic strategies targeting not only malignant, more differentiated GBM tumor cells, but also GBM stem cells that likely contribute to the inevitable recurrence of this disease. The potential phenotypic differences between GBM stem cells and normal NSCs may be subtle, and may in fact be undetectable by currently used clinical tests. Additionally, the source of BTSCs may not be the tumor itself. In at least one study in which a spontaneous mouse model of GBM was used, it was found that tumor-initiating cells might migrate from a separate location within the brain, only to repopulate an area with a favorable microenvironment for tumor growth. ${ }^{53}$ Although the combination of invasiveness, the ability to become migratory, and complexity of cellular composition in human GBMs represents a formidable challenge for effective therapy, the identification of BTSCs provides a rational target for new therapies, because these cells appear to be the tumor-initiating component of GBM.

\section{Complex Heterogeneity and Genetic Mutations}

Numerous genetic mutations have been identified in GBM that have been postulated to contribute to gliomagenesis. Alterations in tumor suppressors are the genetic lesions most commonly found. Retinoblastoma protein, the $R B-1$ gene product, is present ubiquitously and at relatively constant levels throughout the body. ${ }^{1}$ In its dephosphorylated state, this protein functions as a tumor suppressor by sequestering molecules that normally promote progression through the cell cycle. ${ }^{1,40}$ Cell proliferative signals, such as RTK activation through ligand binding, in turn promote phosphorylation of RB protein in the nucleus. The progressive state of phosphorylation causes RB to release bound transcription factors such as E2F proteins, allowing them to drive progression of a cell through the mitotic cell cycle. Thus, RB plays a key role in modulating cell division, largely based on its level of phosphorylation. The $R B$ gene is commonly mutated or deleted in primary human GBM specimens, ${ }^{17}$ thereby implicating RB-mediated cell cycle regulation in the progression of GBM.

Deletion or alteration of the $p 53$ tumor suppressor is present in 25 to $30 \%$ of $\mathrm{GBMs}^{29,35,48}$ and has also been extensively studied in these tumors. ${ }^{22}$ The p53 gene is not commonly expressed at high levels in healthy tissues; its expression is induced by a variety of mechanisms usually implicated in the induction of DNA damage, like that caused by radiation exposure. ${ }^{1,22}$ When present in the cell, $p 53$ pro- motes the transcription and translation of proteins that block progression through the cell cycle, allowing DNA damage to be repaired. ${ }^{27}$ Loss of $p 53$ allows cells with DNA damage to progress through the cell cycle unchecked, perpetuating or compounding the DNA damage. ${ }^{1,22,46}$ Direct mutation, deletion, or loss of expression of $p 53$ is commonly observed in GBMs, as in many other solid tumors. ${ }^{22}$

Furthermore, changes in genes upstream of $p 53$ in this pathway are also commonly found in tumors. Amplification or overexpression of MDM2 occurs in 10 to $15 \%$ of GBMs, resulting in a blunted or absent $p 53$ response, and has in some studies been associated with poor prognosis in patients with GBM. ${ }^{19,38,39,41}$ The MDM2 binds to and sequesters p53, promoting its rapid degradation within the cell, thus preventing transcription of antiproliferative genes. ${ }^{33}$ The MDM2 is in turn regulated by another gene product, human p14ARF (called ARF in this paper); ARF antagonizes MDM2, releasing p53 inhibition to induce cell cycle arrest. ${ }^{37}$

Although $A R F$ is located in the INK4A genomic locus and shares exons 2 and 3 with $p 16^{I N K 4 A}$, it differs in structure and function due to a frameshift caused by splicing from an alternative exon $1 .{ }^{24}$ This second gene encoded by the INK4A locus, $p 16^{I N K 4 A}$, also functions as a tumor suppressor, but with a different mechanism and specificity than ARF. The $\mathrm{p} 16^{\mathrm{INK} 4 \mathrm{~A}}$ locus indirectly regulates $\mathrm{RB}$ by blocking the action of cyclin-dependent kinases, preventing phosphorylation of RB, and maintaining RB-mediated inhibition of cell cycle progression. ${ }^{22}$ Thus, each of the genes encoded by the INK4A locus regulate tumor suppressors, and loss of each has been shown to cause transformation of cells to a malignant phenotype. Therefore, both are considered tumor suppressors and have been implicated in gliomagenesis. Recent studies in mice indicate that ARF may play a more prominent role in gliomagenesis than $\mathrm{p} 16$, although there may be differences in the function of these proteins in mice compared with humans..$^{22,24}$

Separate studies have demonstrated variability of gene expression in different sets of GBMs. ${ }^{16,28,32}$ In particular, tumors expressing the EGFR, which is associated with a particularly poor prognosis, can clearly be identified by microarray expression analysis. ${ }^{32}$ In other studies, investigators have been able to identify subtypes of GBM based on their molecular signature. ${ }^{21,28,32,34}$ Some of these researchers have further demonstrated that overexpression of specific genes correlates with a more favorable prognosis, although the mechanism by which they were overexpressed was not evaluated. In one study, expression analysis was correlated with copy number changes determined using comparative genomic hybridization, and global gene expression changes were observed in genes not associated with chromosomal regions implicated in gene amplification or deletion. ${ }^{34}$ Specifically, copy number changes in genes located on chromosome 10 were associated with global gene expression changes. Interestingly, loss of heterozygosity at the chromosomal location 10q23 is a common feature in GBM.

Chromosomal deletions, mutations, and amplifications all play roles in the development of malignancies, including GBMs. Many GBMs may have common features attributable to these genetic changes. Nevertheless, the circuitous path each individual tumor has followed to reach malignant transformation is likely unique to that tumor and patient, and thus may require therapeutic strategies tailored specifically for each patient and GBM. 


\section{Aberrant Signal Transduction}

In addition to loss of tumor suppressor function, gain of function changes also occur in GBMs and, as in other tumors, often affect signal transduction pathways (Fig. 1). The EGFR is an RTK that is commonly overexpressed in GBMs, and this overexpression typically occurs as a result of gene amplification.,14,52 The EGFR-mediated signal transduction results in activation of a number of downstream pathways including PI3K-AKT and RAS-MAPK, inhibiting apoptosis and driving proliferation. ${ }^{14}$ Alternatively, deletion within the EGFR coding sequence can result in the expression of a truncated, mutant EGFR protein that signals constitutively.

Other mutations common in GBM affect RTKs other than EGFR. Expression of PDGFR $\beta$ as well as its ligand is frequently found in GBMs. ${ }^{31}$ The PDGFR $\beta$ activates RASMAPK and PI3K-AKT as well as JAK-STAT signaling, which is involved in cell survival, proliferation, and differentiation. ${ }^{31}$ Specific dysregulation of RAS-MAPK caused by loss of the NF1 gene in neurofibromatosis Type 1 syndrome has been associated with an increased risk of malignant astrocytomas in affected individuals. ${ }^{53}$ Therefore, the
RAS-MAPK signaling cascade has been presumed to be important for gliomagenesis. Recent evidence indicates, however, that mutant EGFR and overexpressed wild-type EGFR signal preferentially down divergent pathways in GBM; down RAS-MAPK and PI3K-AKT pathways, respectively. ${ }^{52}$

Inducible models of tumorigenesis in mice have yielded findings that particular tumors become dependent on specific mutations, and that repression of the initiating oncogenic stimulus can result in widespread apoptosis and tumor regression. ${ }^{8,10,18,30}$ Targeting of specific molecular defects on which tumor cells rely is the goal of many novel therapeutic strategies (Table 1). Many of these strategies, however, depend on comprehensive mutation or expression analysis. Because RTKs and signaling pathways represent attractive targets for molecular therapeutics, distinguishing the nature of pathway activation in individual tumors will be important for the selection of the appropriate therapeutic modality. Again, the pathway to complete malignant transformation is likely unique in every patient and, although similar pathways are implicated in gliomagenesis by changes in various upstream signaling molecules, the overall mechanisms of pathway activation in

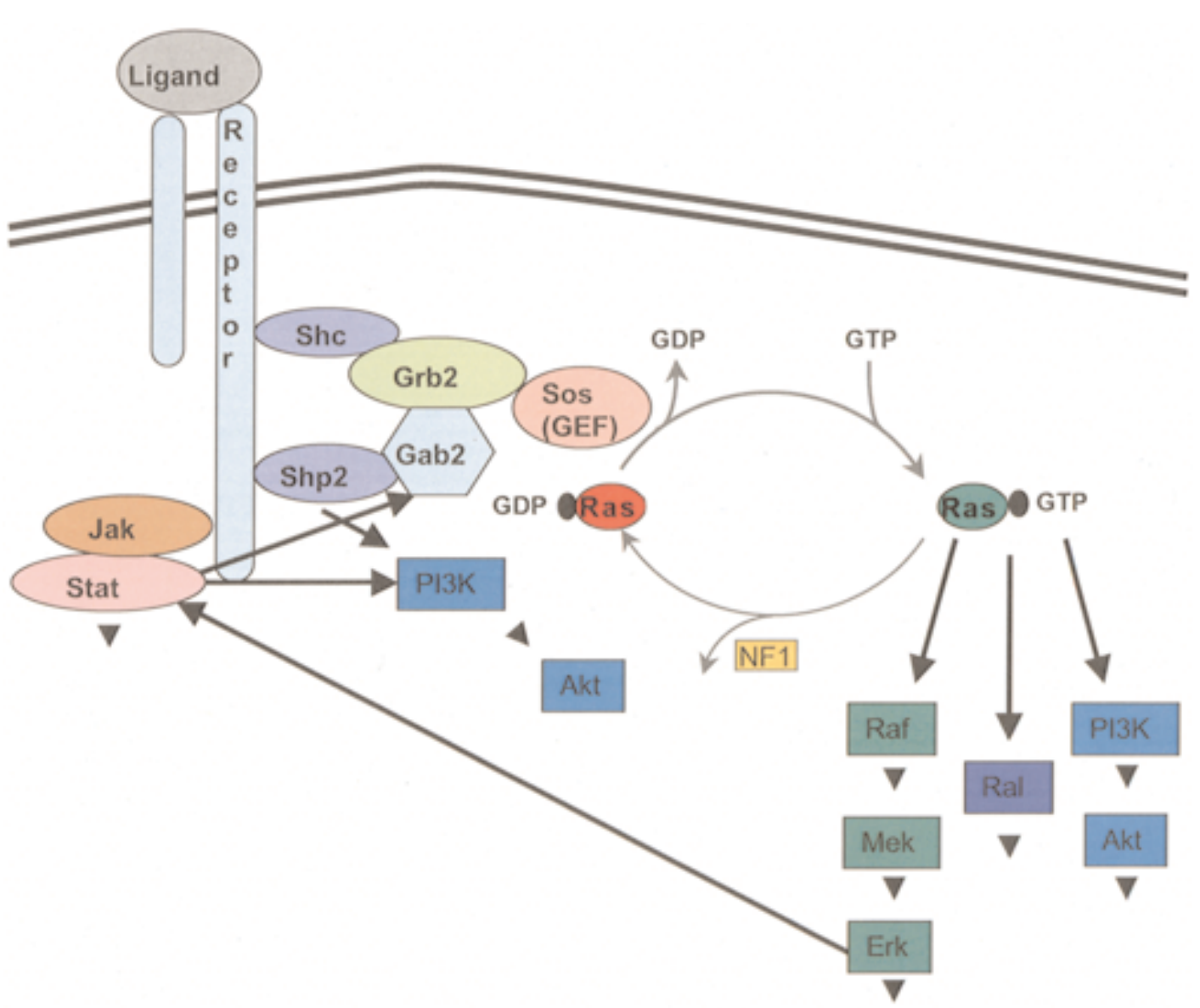

Fig. 1. Schematic depicting signaling with the growth factor receptors through the JAK-STAT and RAS pathways. (Signaling occurs through a number of pathways, including JAK-STAT and RAS-MAPK.) Known interactions between specific effectors are indicated by bold arrows, and arrowheads indicate downstream signaling. Similar schematics could be drawn depicting RTK signaling, but there would be no dependence on JAK activation, because many RTKs can activate STATs directly. Evidence indicates that SHP-2-dependent signaling activates PI3-K or RAS-MAPK differentially from wild-type EGFR or EGFRvIII. GDP = guanosine 5' -diphosphate; GTP = guanosine 5' -triphosphate. 
each tumor may play an important role in the ultimate malignant phenotype. Analysis of the pathways involved in individual tumors should therefore be performed to assist in the selection of therapies that address the unique biology of the specific tumor.

For instance, molecular inhibitors of MDM2 have been recently developed for clinical use. These compounds, called "nutlins," interfere with the interaction between MDM2 and p53, releasing p53 from negative regulation and allowing transcription of cell cycle inhibitory molecules. ${ }^{50}$ Because MDM2 is the second most commonly amplified gene in GBM, use of nutlins in this disease seems a rational therapeutic strategy. Nevertheless, this approach is likely to be most effective only in patients with MDM2 overexpression or low p14 ${ }^{A R F}$ expression. Clinical trials using nutlins as a component of therapy should therefore be designed to include expression and mutation analysis of these two genes as a criterion for patient selection.

\section{Expression Analysis for Rational and Patient-Tailored Therapy}

An increasing body of evidence demonstrates the utility of expression profiling in stratification of patients with GBM in terms of tumor classification and survival. Unfortunately, a marker of survival in GBM is a relative term that belies the fact that less than $1 \%$ of patients survive more than 5 years. ${ }^{25,42}$ Although identification of markers relevant for patient stratification will undoubtedly contribute to improvements in treatment modality, it is important to expand these studies to include the identification of rational therapeutic targets, which seems a more compelling goal in evaluation of GBM at the transcriptional level.

In addition to intertumoral heterogeneity, studies evaluating differential gene expression within the same tumor have demonstrated intratumoral heterogeneity between core GBM tissue and invasive, proliferating peripheral tumor tissue. ${ }^{16}$ Expression differences in one study correlated with differences in the intensity of contrast enhancement on magnetic resonance imaging. ${ }^{15}$ This intratumoral hetero-

TABLE 1

Literature review of currently targeted changes in malignancies*

\begin{tabular}{|c|c|c|}
\hline Authors \& Year & $\begin{array}{l}\text { Phenotypic or } \\
\text { Genetic Change }\end{array}$ & Targeted Therapy \\
\hline Carroll, et al., 1997 & $\begin{array}{l}\text { PDGFR/PDGF over- } \\
\text { expression }\end{array}$ & $\begin{array}{l}\text { imatinib mesylate } \\
\text { (Gleevec) }\end{array}$ \\
\hline Debinski, et al., 1999 & IL13R overexpression & immunotoxins \\
\hline Husain, et al., 2001 & IL13R overexpression & immunotoxins \\
\hline Traxler, et al., 2001 & $\begin{array}{l}\text { EGFR amplification, } \\
\text { EGFRvIII }\end{array}$ & $\begin{array}{c}\text { ZD1839 (IRESSA), } \\
\text { OSI-774, PK1166, } \\
\text { CI1033, EKB569 }\end{array}$ \\
\hline Shawver, et al., 2002 & $\begin{array}{l}\text { EGFR amplification, } \\
\text { EGFRvIII }\end{array}$ & $\begin{array}{c}\text { ZD1839 (IRESSA), } \\
\text { OSI-774, PK1166, } \\
\text { CI1033, EKB569 }\end{array}$ \\
\hline Ferrara, et al., 2004 & VEGF overexpression & antiangiogenesis \\
\hline Vassilev, et al., 2004 & $\begin{array}{l}\text { ARF loss } \\
M D M 2 \text { amplification }\end{array}$ & $\begin{array}{l}\text { nutlins } \\
\text { nutlins }\end{array}$ \\
\hline Chang, et al., 2005 & PTEN loss & CCI-779, RAD001 \\
\hline Galanis, et al., 2005 & PTEN loss & CCI-779, RAD001 \\
\hline Goudar, et al., 2005 & PTEN loss & CCI-779, RAD001 \\
\hline Ohlfest, et al., 2005 & VEGF overexpression & antiangiogenesis \\
\hline
\end{tabular}

* VEGF = vascular endothelial growth factor. geneity again highlights the need for multifaceted modalities addressing all aspects of the biology of GBMs. These studies demonstrate the utility of expression profiling in the stratification of patients as well as the selection of targeted molecular and gene therapies used for successful treatment of this disease.

The identification of BTSCs as candidates for the true tumor-initiating cells poses an interesting challenge for conducting routine, high-throughput gene expression analysis of GBMs to tailor therapy to rational targets. It is vital to kill the BTSCs as well as the more differentiated tumor cells to achieve the maximum antitumor effect. Accordingly, it may be critical to purify CD133-positive BTSCs for expression analysis to determine which class of drugs is most likely to kill these cells. In addition, expression analysis could be conducted in the CD133-negative tumor cells to identify a second drug tailored for their elimination.

\section{CONCLUSIONS}

In-depth expression analysis with respect to mutation status has not been performed with GBMs to the same extent as with other cancers. ${ }^{2,26,49}$ With the development of molecular inhibitors targeting multiple pathways implicated in gliomagenesis, the combination of expression analysis and mutation status may be the next step in enabling the development of therapeutic modalities better tailored for individual disease characteristics. It is clear that the appropriate drugs for GBM will not be the same for all patients, and that choosing the best drug may require routine expression analysis. Therefore, expression analysis may be most useful as a standard clinical test performed before drug therapy commences to choose rationally patient-tailored drugs that target the appropriate molecular pathways. For instance, expression analysis could be conducted immediately after resection in multiple biopsy samples of core and invasive regions of the tumor, perhaps identified before resection on magnetic resonance imaging.

Implementing expression analysis as a standard clinical test, however, will require significant changes in the way clinicians approach GBM treatment, as well as the development of substantial infrastructure for rapid, high-throughput expression and mutation analysis of tumor tissue. The challenge of interpreting large data sets generated from routine, high-throughput array analysis from individual tumors will require an expansion of expertise from academic, researchoriented studies to wider clinical practice. Despite these obstacles, it is vital to take into account the genetic mutations present in the patient's individual tumor before the use of drugs that target molecular pathways can ever be expected to achieve maximal clinical efficacy. Furthermore, it is important to consider that GBMs are composed of a group of related but genetically distinct tumor cells, containing both BTSCs and differentiated tumor cells, and capitalize on this knowledge by using therapies that target all populations present in the tumor.

\section{References}

1. Alberts B, Bray D, Lewis J, et al: Molecular Biology of the Cell, ed 3. New York: Garland Publishing, 1994

2. Bullinger L, Dohner K, Bair E, et al: Use of gene-expression profiling to identify prognostic subclasses in adult acute myeloid leukemia. N Engl J Med 350:1605-1616, 2004 
3. Carroll M, Ohno-Jones S, Tamura S, et al: CGP 57148, a tyrosine kinase inhibitor, inhibits the growth of cells expressing BCRABL, TEL-ABL, and TEL-PDGFR fusion proteins. Blood 90:4947-4952, 1997

4. Chakravarti A, Seiferheld W, Tu X, et al: Immunohistochemically determined total epidermal growth factor receptor levels not of prognostic value in newly diagnosed glioblastoma multiforme: report from the Radiation Therapy Oncology Group. Int J Radiat Oncol Biol Phys 62:318-327, 2005

5. Chang SM, Wen P, Cloughesy T, et al: Phase II study of CCI779 in patients with recurrent glioblastoma multiforme. Invest New Drugs 23:357-361, 2005

6. Daumas-Duport C, Scheithauer B, O'Fallon J, et al: Grading of astrocytomas. A simple and reproducible method. Cancer 62: 2152-2165, 1988

7. Debinski W, Gibo DM, Hulet SW, et al: Receptor for interleukin 13 is a marker and therapeutic target for human highgrade gliomas. Clin Cancer Res 5:985-990, 1999

8. Felsher DW, Bishop JM: Reversible tumorigenesis by MYC in hematopoietic lineages. Mol Cell 4:199-207, 1999

9. Ferrara N, Hillan KJ, Gerber HP, et al: Discovery and development of bevacizumab, an anti-VEGF antibody for treating cancer. Nat Rev Drug Discov 3:391-400, 2004

10. Fisher GH, Wellen SL, Klimstra D, et al: Induction and apoptotic regression of lung adenocarcinomas by regulation of a KRas transgene in the presence and absence of tumor suppressor genes. Genes Dev 15:3249-3262, 2001

11. Freese A, O'Rourke D, Judy K, et al: The application of 5-bromodeoxyuridine in the management of CNS tumors. J Neurooncol 20:81-95, 1994

12. Galanis E, Buckner JC, Maurer MJ, et al: Phase II trial of temsirolimus (CCI-779) in recurrent glioblastoma multiforme: a North Central Cancer Treatment Group Study. J Clin Oncol 23:5294-5304, 2005

13. Goudar RK, Shi Q, Hjelmeland MD, et al: Combination therapy of inhibitors of epidermal growth factor receptor/vascular endothelial growth factor receptor 2 (AEE788) and the mammalian target of rapamycin (RAD001) offers improved glioblastoma tumor growth inhibition. Mol Cancer Ther 4:101-112, 2005

14. Grunwald V, Hidalgo M: Developing inhibitors of the epidermal growth factor receptor for cancer treatment. J Natl Cancer Inst 95:851-867, 2003

15. Hobbs SK, Shi G, Homer R, et al: Magnetic resonance imageguided proteomics of human glioblastoma multiforme. J Magn Reson Imaging 18:530-536, 2003

16. Hoelzinger DB, Mariani L, Weis J, et al: Gene expression profile of glioblastoma multiforme invasive phenotype points to new therapeutic targets. Neoplasia 7:7-16, 2005

17. Hu J, Jiang C, Ng HK, et al: Genome-wide allelotype study of primary glioblastoma multiforme. Chin Med J (Engl) 116: 577-583, 2003

18. Huettner CS, Zhang P, Van Etten RA, et al: Reversibility of acute B-cell leukaemia induced by BCR-ABL1. Nat Genet 24: 57-60, 2000

19. Hunter SB, Abbott K, Varma VA, et al: Reliability of differential PCR for the detection of EGFR and MDM2 gene amplification in DNA extracted from FFPE glioma tissue. J Neuropathol Exp Neurol 54:57-64, 1995

20. Husain SR, Joshi BH, Puri RK: Interleukin-13 receptor as a unique target for anti-glioblastoma therapy. Int J Cancer 92: $168-175,2001$

21. Kim S, Dougherty ER, Shmulevich I, et al: Identification of combination gene sets for glioma classification. Mol Cancer Ther 1:1229-1236, 2002

22. Krug U, Ganser A, Koeffler HP: Tumor suppressor genes in normal and malignant hematopoiesis. Oncogene 21:3475-3495, 2002

23. Kuratsu J, Itoyama Y, Uemura S, et al: [Regrowth patterns of glioma-cases of glioma regrew away from the original tumor.] Gan No Rinsho 35:1255-1260, 1989 (Jpn)

24. Kusy S, Larsen CJ and Roche J: p14ARF, p15INK4b and p16INK4a methylation status in chronic myelogenous leukemia. Leuk Lymphoma 45:1989-1994, 2004

25. Lacroix M, Abi-Said D, Fourney DR, et al: A multivariate analysis of 416 patients with glioblastoma multiforme: prognosis, extent of resection, and survival. J Neurosurg 95:190-198, 2001

26. Lapointe J, Li C, Higgins JP, et al: Gene expression profiling identifies clinically relevant subtypes of prostate cancer. Proc Natl Acad Sci U S A 101:811-816, 2004

27. Lewin B: Genes VII. New York: Oxford University Press, 1999

28. Liang Y, Diehn M, Watson N, et al: Gene expression profiling reveals molecularly and clinically distinct subtypes of glioblastoma multiforme. Proc Natl Acad Sci U S A 102:5814-5819, 2005

29. Louis DN, Holland EC, Cairncross JG: Glioma classification: a molecular reappraisal. Am J Pathol 159:779-786, 2001

30. Marinkovic D, Marinkovic T, Mahr B, et al: Reversible lymphomagenesis in conditionally c-MYC expressing mice. Int J Cancer 110:336-342, 2004

31. Mischel PS, Cloughesy TF: Targeted molecular therapy of GBM. Brain Pathol 13:52-61, 2003

32. Mischel PS, Shai R, Shi T, et al: Identification of molecular subtypes of glioblastoma by gene expression profiling. Oncogene 22:2361-2373, 2003

33. Momand J, Zambetti GP, Olson DC, et al: The mdm-2 oncogene product forms a complex with the $\mathrm{p} 53$ protein and inhibits p53-mediated transactivation. Cell 69:1237-1245, 1992

34. Nigro JM, Misra A, Zhang L, et al: Integrated array-comparative genomic hybridization and expression array profiles identify clinically relevant molecular subtypes of glioblastoma. Cancer Res 65:1678-1686, 2005

35. Ohgaki H, Schauble B, zur Hausen A, et al: Genetic alterations associated with the evolution and progression of astrocytic brain tumors. Virchows Arch 427:113-118, 1995

36. Ohlfest JR, Demorest ZL, Motooka Y, et al: Combinatorial antiangiogenic gene therapy by nonviral gene transfer using the sleeping beauty transposon causes tumor regression and improves survival in mice bearing intracranial human glioblastoma. Mol Ther:[Epub ahead of print], 2005

37. Pomerantz J, Schreiber-Agus N, Liegeois NJ, et al: The Ink4a tumor suppressor gene product, p19Arf, interacts with MDM2 and neutralizes MDM2's inhibition of p53. Cell 92:713-723, 1998

38. Rainov NG, Dobberstein KU, Bahn H, et al: Prognostic factors in malignant glioma: influence of the overexpression of oncogene and tumor-suppressor gene products on survival. J Neurooncol 35:13-28, 1997

39. Reifenberger G, Reifenberger J, Ichimura K, et al: Amplification at 12q13-14 in human malignant gliomas is frequently accompanied by loss of heterozygosity at loci proximal and distal to the amplification site. Cancer Res 55:731-734, 1995

40. Sauerbrey A, Stammler G, Zintl F, et al: Expression of the retinoblastoma tumor suppressor gene (RB-1) in acute leukemia. Leuk Lymphoma 28:275-283, 1998

41. Schiebe M, Ohneseit P, Hoffmann W, et al: Analysis of mdm2 and p53 gene alterations in glioblastomas and its correlation with clinical factors. J Neurooncol 49:197-203, 2000

42. Scott JN, Rewcastle NB, Brasher PM, et al: Long-term glioblastoma multiforme survivors: a population-based study. Can J Neurol Sci 25:197-201, 1998

43. Shawver LK, Slamon D, Ullrich A: Smart drugs: tyrosine kinase inhibitors in cancer therapy. Cancer Cell 1:117-123, 2002

44. Silbergeld DL, Chicoine MR: Isolation and characterization of human malignant glioma cells from histologically normal brain. J Neurosurg 86:525-531, 1997 


\section{S. M. Wiesner, A. Freese, and J. R. Ohlfest}

45. Singh SK, Hawkins C, Clarke ID, et al: Identification of human brain tumor initiating cells. Nature 432:396-401, 2004

46. Strachan T, Read AP: Human Molecular Genetics 1, ed 2. New York: Wiley, 1999

47. Traxler P, Bold G, Buchdunger E, et al: Tyrosine kinase inhibitors: from rational design to clinical trials. Med Res Rev 21: 499-512, 2001

48. Ushio Y, Tada K, Shiraishi S, et al: Correlation of molecular genetic analysis of p53, MDM2, p16, PTEN, and EGFR and survival of patients with anaplastic astrocytoma and glioblastoma. Front Biosci 8: 281-e288, 2003

49. Valk PJ, Verhaak RG, Beijen MA, et al: Prognostically useful gene-expression profiles in acute myeloid leukemia. $\mathbf{N}$ Engl J Med 350:1617-1628, 2004

50. Vassilev LT, Vu BT, Graves B, et al: In vivo activation of the p53 pathway by small-molecule antagonists of MDM2. Science 303:844-848, 2004
51. Yuan X, Curtin J, Xiong Y, et al: Isolation of cancer stem cells from adult glioblastoma multiforme. Oncogene 23:9392-9400, 2004

52. Zhan Y, O'Rourke DM: SHP-2-dependent mitogen-activated protein kinase activation regulates EGFRvIII but not wild-type epidermal growth factor receptor phosphorylation and glioblastoma cell survival. Cancer Res 64:8292-8298, 2004

53. Zhu Y, Guignard F, Zhao D, et al: Early inactivation of p53 tumor suppressor gene cooperating with NF1 loss induces malignant astrocytoma. Cancer Cell 8:119-130, 2005

Manuscript received September 14, 2005.

Accepted in final form September 23, 2005.

Address reprint requests to: John Ohlfest, Ph.D., Institute of Human Genetics, Department of Neurosurgery, 4-122 Moos Tower, MMC 206, Minneapolis, Minnesota 55422. email: ohlfe001@umn.edu. 